2. Chong Y, Shimoda S, Yakushiji H, et al. Community spread of extendedspectrum $\beta$-lactamase-producing Escherichia coli, Klebsiella pneumoniae and Proteus mirabilis: a long-term study in Japan. J Med Microbiol 2013;62:1038-1043.

3. Kimura Y, Fukuda H, Hayakawa K, et al. Longitudinal trends of and factors associated with inappropriate antibiotic prescribing for non-bacterial acute respiratory tract infection in Japan: a retrospective claims database study, 2012-2017. PLoS One 2019;14:1-15.

4. Tsuzuki S, Kimura Y, Ishikane M, Kusama Y, Ohmagari N. Cost of inappropriate antimicrobial use for upper respiratory infection in Japan. BMC Health Serv Res 2020;20(1):153.
5. Muraki Y, Kitamura M, Maeda Y, et al. Nationwide surveillance of anti consumption and resistance to Pseudomonas aeruginosa isolates at 203 Japanese hospitals in 2010. Infection 2013;41:415-423.

6. Tanimura H, Uchiyama K, Onishi $\mathrm{H}$, et al. Study of the absorption of cefcapene pivoxil in patients with infectious disease and soft stool or diarrhea. J Infect Chemother 2003;9:75-82.

7. Sádaba B, Azanza JR, Quetglas EG, et al. Pharmacokinetic/pharmacodynamic serum and urine profile of cefditoren following single-dose and multiple twice- and thrice-daily regimens in healthy volunteers: a phase I study. Rev Esp Quimioter 2007;20:51-60.

\title{
Rational allocation of coronavirus disease 2019 (COVID-19) vaccines to healthcare personnel and patients: A role for antimicrobial stewardship programs?
}

\author{
Priya Nori MD ${ }^{1}$ (1) Payal K. Patel MD, $\mathrm{MPH}^{2}$ and Michael P. Stevens MD, MPH ${ }^{3}$ \\ ${ }^{1}$ Division of Infectious Diseases, Department of Medicine, Montefiore Medical Center, Albert Einstein College of Medicine, Bronx, New York, ${ }^{2}$ Infectious Diseases \\ Section, Ann Arbor Veterans' Affairs Medical Center, Ann Arbor, Michigan and ${ }^{3}$ Healthcare Infection Prevention Department, Virginia Commonwealth University \\ Health System, Richmond, Virginia
}

To the Editor-Vaccine allocation planning is not traditionally considered a core activity of antimicrobial stewardship programs (ASPs). ${ }^{1,2}$ However, ASP physicians and pharmacists are well suited to participate in health-system-wide coronavirus disease 2019 (COVID-19) vaccination efforts given their expertise, programmatic infrastructure, institutional leadership, and trust within their organizations. Although ASPs have played a central role in the COVID-19 response pertaining to novel therapeutics, 3,4 little has been published on ASP involvement in COVID-19 vaccine allocation planning. A PubMed search for "antimicrobial stewardship and severe acute respiratory coronavirus virus 2 (SARS-CoV-2) vaccine" and "antimicrobial stewardship and COVID-19 vaccine" on December 2, 2020, returned no relevant results. Given the rapid expansion of their roles during the pandemic, ASPs may be asked to contribute to health system COVID-19 vaccine allocation planning. ASP contributions to similar institutional efforts predate the pandemic. For instance, ASPs hold important roles in institutional pharmacy and therapeutics committees for formulary addition of new vaccines (eg, new recombinant zoster and meningococcal vaccines). Historically, some ASPs were integrated into 2009 H1N1 influenza pandemic response efforts, participating in vaccine planning and the development of antiviral treatment guidelines. ${ }^{5}$

Since the COVID-19 pandemic began, ASPs have developed significant experience in designing rational allocation systems for novel therapeutics such as remdesivir, ${ }^{4}$ which is now incorporated into ASP preauthorization paradigms throughout the country. ASP pharmacists have particular expertise in preparation,

\footnotetext{
Author for correspondence: Priya Nori, E-mail: pnori@montefiore.org

Cite this article: Nori P, Patel PK, and Stevens MP. (2022). Rational allocation of coronavirus disease 2019 (COVID-19) vaccines to healthcare personnel and patients: A role for antimicrobial stewardship programs?. Infection Control \& Hospital Epidemiology, 43: 263-265, https://doi.org/10.1017/ice.2020.1393
}

transport, and storage required for specific COVID-19 vaccine products. Extreme temperature requirements pose a major challenge for community hospitals and nursing facilities in rural areas without access to medical-grade deep freezers. ${ }^{6}$ ASPs can develop coordinated vaccine distribution systems from a centralized location within health systems to distant facilities. ASPs frequently interface with health information technology departments to design electronic medical record add-ons for stewardship functions, which can be harnessed to create a streamlined order set for all available COVID-19 vaccines and templates for mandatory documentation and reporting required by state health departments.

ASPs have also become a trusted resource for accurate interpretation and dissemination of published data and society guidelines on SARS-CoV-2 therapeutics, such as remdesivir, convalescent plasma, and monoclonal antibodies. Applying this concept to vaccines, ASPs can work with institutional leaders to review data from COVID-19 vaccine trials and adapt recommendations of the Advisory Committee on Immunization Practices (ACIP) on ethical vaccine allocation to their health systems, with the goals of maximizing benefits and minimizing harms, promoting justice and transparency, and mitigating health inequities. ${ }^{7}$ ASPs can also help health systems quickly adapt to evolving guidance from public health authorities in terms of initial target vaccination groups. ${ }^{7}$ Healthcare workers at greatest risk of exposure as well as those caring for the most vulnerable patients will be prioritized in phase 1 of vaccination. ${ }^{7}$ Partnership with local skilled nursing facilities (SNFs) may be required to bolster their resident and associate vaccination efforts. ASP pharmacists and physicians are well suited to provide expertise pertaining to vaccine education, preparation, storage, and monitoring and reporting side effects.

Although ASPs are assets to vaccine allocation planning, many challenges remain. Due to limited initial vaccine doses, a

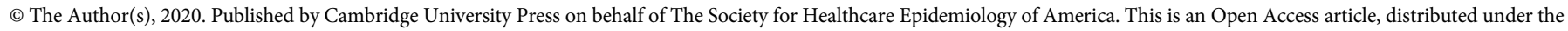
terms of the Creative Commons Attribution licence (http://creativecommons.org/licenses/by/4.0/), which permits unrestricted re-use, distribution, and reproduction in any medium, provided the original work is properly cited. 


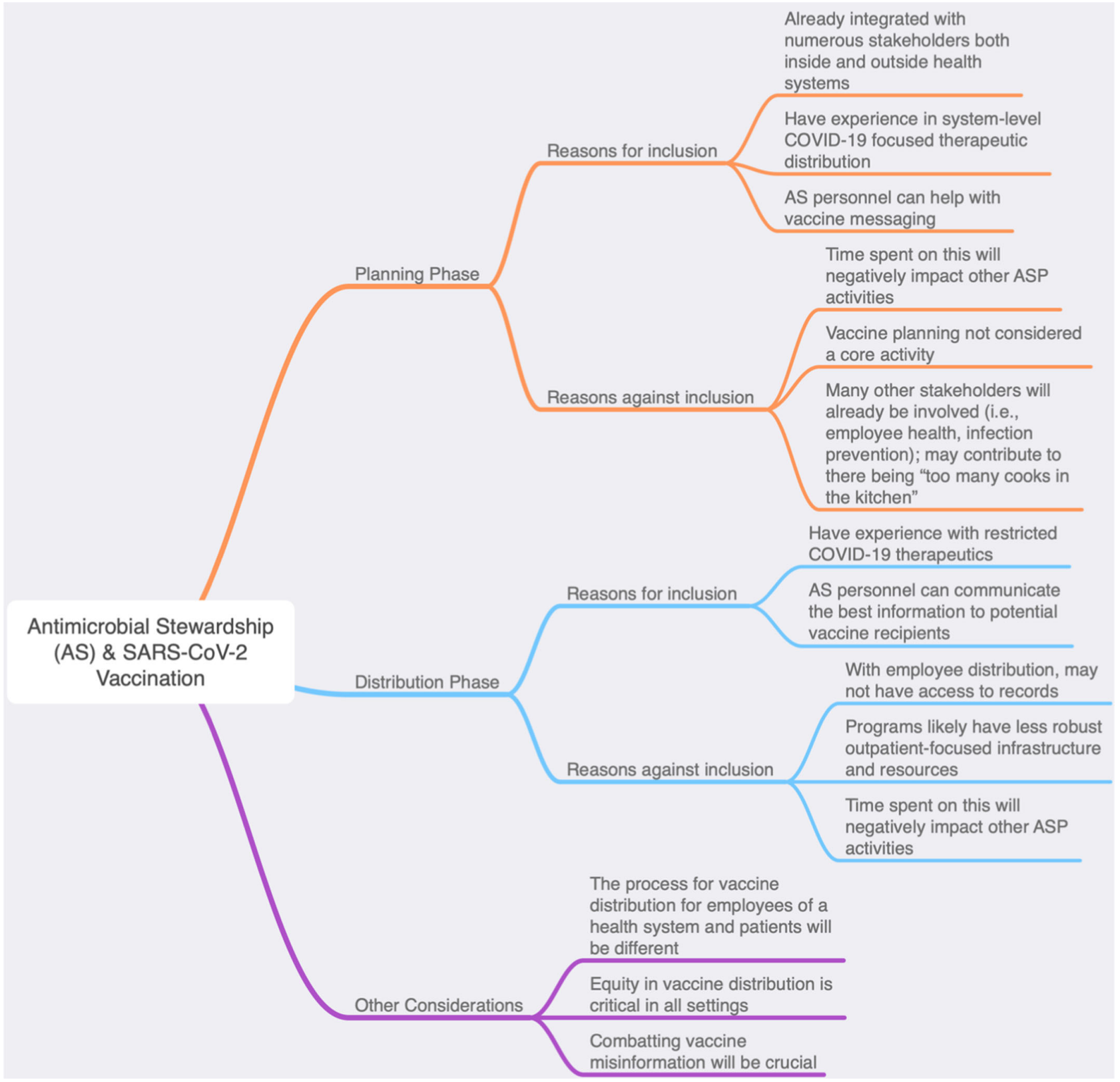

Fig. 1. Antimicrobial stewardship (AS) and SARS-CoV-2 vaccination.

preauthorization and careful tracking mechanism is ideal; however, ASP preauthorization is primarily limited to antimicrobials used in the inpatient setting, and vaccines are outside the scope of this paradigm. Additionally, employee health records systems can exist separately from patient electronic medical records systems, and close coordination with hospital occupational health programs will be required. Another major hurdle is an "infodemic" of COVID-19 misinformation. ${ }^{8}$ However, as mentioned above, ASPs are a trusted source of information, and they have considerable experience educating both HCWs and patients on appropriate antimicrobial use. This skill will prove useful when addressing hesitancy concerning novel vaccines with only short-term safety data. For instance, ASPs can reinforce Centers for Disease Control and Prevention recommendations regarding the role of COVID-19 vaccination in individuals with past infection and evidence of natural immunity. ${ }^{9,10}$ Patient and staff education are routinely conducted by ASP staff. ${ }^{11}$

Notably, ASP involvement in COVID-19 vaccination efforts will require a significant investment of effort at a time when ASPs are already tasked with other important pandemic-related roles (eg, developing outpatient monoclonal antibody infusion programs). ${ }^{12}$ This effort will naturally come at the expense of other ASP activities such as ensuring appropriate perioperative prophylaxis for elective surgical procedures, which may continue at certain hospitals to financially support institutions. Health systems will need to invest in ASPs in terms of dedicated effort and resources to achieve desired outcomes during the pandemic. To mitigate potential loss of other critical ASP functions, tracking, and reporting of vaccinations should be diverted to other hospital personnel after initial ASP-lead education and allocation planning. 


\section{Acknowledgments.}

Financial support. No financial support was provided relevant to this article.

Conflicts of interest. All authors report no conflicts of interest relevant to this article.

\section{References}

1. Dellit TH, Owens RC, McGowan JE, et al. Infectious Diseases Society of America and the Society for Healthcare Epidemiology of America guidelines for developing an institutional program to enhance antimicrobial stewardship. Clin Infect Dis 2007;44:159-177.

2. Core elements of hospital antibiotic stewardship programs. Centers for Disease Control and Prevention (CDC) website. https://www.cdc. gov/antibiotic-use/core-elements/hospital.html. Published 2020. Accessed November 16, 2020.

3. Stevens MP, Patel PK, Nori P. Involving antimicrobial stewardship programs in COVID-19 response efforts: all hands on deck. Infect Control Hosp Epidemiol 2020;41:744-745.

4. Mazdeyasna H, Nori P, Patel P. et al. Antimicrobial stewardship at the core of COVID-19 response efforts: implications for sustaining and building programs. Curr Infect Dis Rep 2020;22(9):23.

5. Ahmad H, Guo Y, Yaghdjian V, et al. Hospital emergency response to novel influenza A (H1N1) pandemic in a large New York City hospital: an opportunity for antimicrobial stewardship. Hosp Pharm 2012;47:532-537.
6. Crommelin DJA, Volkin DB, Hoogendoorn KH, Lubiniecki AS, Jiskoot W. The science is there: key considerations for stabilizing viral vector-based COVID-19 vaccines. J Pharm Sci 2020. doi: 10.1016/j.xphs.2020.11.015

7. ACIP presentation slides: December 2020 meeting, December 1, 2020. Coronavirus disease (COVID-19) vaccines. Centers for Disease Control and Prevention (CDC) website. https:/www.cdc.gov/vaccines/acip/meetings/ slides-2020-12.html. Published 2020. Accessed December 2, 2020.

8. Biasio LR, Bonaccorsi G, Lorini C, Pecorelli S. Assessing COVID-19 vaccine literacy: a preliminary online survey. Hum Vaccin Immunother 2020. doi: 10.1080/21645515.2020.1829315.

9. Lazarus JV, Ratzan SC, Palayew A, et al. A global survey of potential acceptance of a COVID-19 vaccine. Nat Med 2020. doi: 10.1038/s41591020-1124-9.

10. Facts about COVID-19 vaccines. Centers for Disease Control and Prevention (CDC) website. https://www.cdc.gov/coronavirus/2019-ncov/ vaccines/vaccine-benefits/facts.html?CDC_AA_refVal=https\%3A\%2F\% 2Fwww.cdc.gov\%2Fcoronavirus\%2F2019-ncov\%2Fvaccines\%2Fabout-vaccines \%2Fvaccine-myths.html. Published 2020. Accessed December 3, 2020.

11. Antimicrobial stewardship requirements for hospitals. The Joint Commission website. https://www.jointcommission.org/-/media/deprecated-unorganized/ imported-assets/tjc/system-folders/assetmanager/adhoc_hap_abx_r3_201608 22pdf.pdf?db=web\&hash=10A09E9017D5576E6E101FFB789C8336. Published 2020. Accessed December 2, 2020.

12. Patel PK, Nori P, Stevens MP. Antimicrobial stewardship and bamlanivimab: opportunities for outpatient preauthorization? Infect Control Hosp Epidemiol 2020. doi: 10.1017/ice.2020.1343.

\title{
"Would you like a flu shot with your order?"-A coronavirus disease 2019 (COVID-19) pandemic drive-through response to address delayed pediatric immunization in Detroit, Michigan
}

\author{
Eric J. McGrath $\mathrm{MD}^{1}$ (1) , Donia Dalal MPH${ }^{2}$, Lynn Smitherman $\mathrm{MD}^{1}$, Sharon Marshall MD ${ }^{1}$, Christopher Youngman MD $^{1}$, \\ Charles J. Barone MD $^{3}$, Herman Gray MD ${ }^{1}$, Najibah Rehman MD $^{2}$ and Elizabeth Secord MD ${ }^{1}$ \\ ${ }^{1}$ Department of Pediatrics, Wayne State University School of Medicine, Detroit, Michigan, ${ }^{2}$ Detroit Health Department, Detroit, Michigan and ${ }^{3}$ Department of \\ Pediatrics, Henry Ford Hospital, Detroit, Michigan
}

To the Editor-Since the coronavirus disease 2019 (COVID-19) pandemic and associated "shelter in place" orders in the spring of 2020 , numerous children in the United States, ${ }^{1}$ but especially in Michigan, ${ }^{2}$ have not received the recommended immunizations. Furthermore, baseline Michigan vaccination rates have been low. Here, we describe an effort to address this public health crisis within the COVID-19 crisis.

A "drive-through" immunization fair ${ }^{3-6}$ was held Saturday, October 10,2020 , in which parents and their children (aged 6 weeks to 18 years) stayed in their vehicles and all participants $>2$ years old wore required facemasks. The Wayne Pediatrics (WP) clinical group, affiliated with Wayne State University (WSU) School of Medicine (SOM), collaborated with the Detroit Health Department (DHD) to offer the event. Parents were encouraged to call the DHD to schedule a drive-through

\footnotetext{
Author for correspondence: Eric J. McGrath, E-mail: emcgrath@med.wayne.edu Cite this article: McGrath EJ, et al. (2022). "Would you like a flu shot with your order?"-A coronavirus disease 2019 (COVID-19) pandemic drive-through response to address delayed pediatric immunization in Detroit, Michigan. Infection Control \& Hospital Epidemiology, 43: 265-266, https://doi.org/10.1017/ice.2020.1410
}

appointment before the day of the event, but patients who showed up to the event without an appointment were also seen. Before the event, local families were informed about the event through advertising with bulk mailings of postcards, by social media, by e-mail alerts to community partners, and by other widespread marketing publicity.

Routine vaccines from the 2020 pediatric schedule ${ }^{7}$ were offered to participants due for immunization or requesting influenza vaccine. Immunizations were supplied by the DHD. The WP clinic-building parking lot was used for one-way traffic flow (Fig. 1). Henry Ford Pediatrics donated their pediatric mobile vehicle and professional driver for the event. Older children could receive vaccines in their deltoid or shoulder though their vehicle window, and the mobile unit was used for privacy for infants and very young children vaccinates in the upper thigh.

The DHD dedicated staff members included an immunization coordinator, 2 nurses, 2 patient navigators, and 2 registration staff. The WP clinic staff volunteers included a nurse, 4 medical assistants, the clinical manager, a clinical supervisor, 2 medical secretaries, and 6 physicians. Each completed a 4.5-hour shift for the 12-hour event. 